\title{
Three-dimensional architecture of the whole human soleus muscle in vivo
}

\author{
Bart Bolsterlee $^{\text {Corresp., }}{ }^{1,2}$ ， Taija M Finni ${ }^{3}$ ，Arkiev D'Souza ${ }^{1,2}$ ， Junya Eguchi ${ }^{1,2}$ ， Elizabeth C Clarke ${ }^{4}$, Robert D \\ Herbert ${ }^{1,2}$ \\ 1 Neuroscience Research Australia, Sydney, New South Wales, Australia \\ 2 University of New South Wales, Sydney, New South Wales, Australia \\ 3 Faculty of Sport and Health Sciences, University of Jyväskylä, Jyväskylä, Finland \\ 4 Murray Maxwell Biomechanics Laboratory, Institute for Bone and Joint Research, Kolling Institute of Medical Research, Sydney Medical School, University \\ of Sydney, Sydney, New South Wales, Australia \\ Corresponding Author: Bart Bolsterlee \\ Email address: b.bolsterlee@neura.edu.au
}

Background. Most data on the architecture of the human soleus muscle have been obtained from cadaveric dissection or two-dimensional 2D ultrasound imaging. We present the first comprehensive, quantitative study on the three-dimensional anatomy of the human soleus muscle in vivo using diffusion tensor imaging (DTI) techniques.

Methods. We report three-dimensional fascicle lengths, pennation angles, fascicle curvatures, physiological cross-sectional area and volume in four compartments of the soleus at ankle joint angles of $69 \pm 12^{\circ}$ (plantarflexion, short muscle length; average \pm SD across subjects) and $108 \pm 7^{\circ}$ (dorsiflexion, long muscle length) of six healthy young adults. Microdissection and three-dimensional digitisation on two cadaveric muscles corroborated the compartmentalised structure of the soleus, and confirmed the validity of DTI-based muscle fascicle reconstructions.

Results. The posterior compartments of the soleus comprised $80 \pm 5 \%$ of the total muscle volume (356 $\left.\pm 58 \mathrm{~cm}^{3}\right)$. At the short muscle length, the average fascicle length, pennation angle and curvature was 37 $\pm 8 \mathrm{~mm}, 31 \pm 3^{\circ}$ and $17 \pm 4 / \mathrm{m}$, respectively. We did not find differences in fascicle lengths between compartments. However, pennation angles were on average $12^{\circ}$ larger $(p<0.01)$ in the posterior compartments than in the anterior compartments. For every centimetre that the muscle-tendon unit lengthened, fascicle lengths increased by $3.7 \pm 0.8 \mathrm{~mm}$, pennation angles decreased by $-3.2 \pm 0.9^{\circ}$ and curvatures decreased by $-2.7 \pm 0.8 / \mathrm{m}$. Fascicles in the posterior compartments rotated almost twice as much as in the anterior compartments during passive lengthening.

Discussion. The homogeneity in fascicle lengths and inhomogeneity in pennation angles of the soleus may indicate a functionally different role for the anterior and posterior compartments. The data and techniques presented here demonstrate how DTI can be used to obtain detailed, quantitative measurements of the anatomy of complex skeletal muscles in living humans. 


\section{Three-dimensional architecture of the whole human soleus muscle in vivo}

2

3

4 Bart Bolsterlee ${ }^{1,2}$, Taija Finni ${ }^{3}$, Arkiev D'Souza ${ }^{1,2}$, Junya Eguchi ${ }^{1,2}$, Elizabeth C Clarke ${ }^{4}$, Robert D Herbert ${ }^{1,2}$

5 1) Neuroscience Research Australia, Sydney NSW, Australia

6 2) University of New South Wales, Sydney NSW, Australia

7 3) Neuromuscular Research Center, Faculty of Sport and Health Sciences, University of Jyväskylä,

$8 \quad$ Finland

9 4) Murray Maxwell Biomechanics Laboratory, Institute for Bone and Joint Research, Kolling Institute of Medical Research, Sydney Medical School, University of Sydney, Sydney NSW, Australia

14 Corresponding author:

Dr Bart Bolsterlee 


\section{Abstract}

Background. Most data on the architecture of the human soleus muscle have been obtained from cadaveric dissection or two-dimensional 2D ultrasound imaging. We present the first comprehensive, quantitative study on the three-dimensional anatomy of the human soleus muscle in vivo using diffusion tensor imaging (DTI) techniques.

Methods. We report three-dimensional fascicle lengths, pennation angles, fascicle curvatures, physiological cross-sectional area and volume in four compartments of the soleus at ankle joint angles of $69 \pm 12^{\circ}$ (plantarflexion, short muscle length; average \pm SD across subjects) and $108 \pm 7^{\circ}$ (dorsiflexion, long muscle length) of six healthy young adults. Microdissection and three-dimensional digitisation on two cadaveric muscles corroborated the compartmentalised structure of the soleus, and confirmed the validity of DTI-based muscle fascicle reconstructions.

Results. The posterior compartments of the soleus comprised $80 \pm 5 \%$ of the total muscle volume (356 \pm $58 \mathrm{~cm}^{3}$ ). At the short muscle length, the average fascicle length, pennation angle and curvature was $37 \pm$ $8 \mathrm{~mm}, 31 \pm 3^{\circ}$ and $17 \pm 4 / \mathrm{m}$, respectively. We did not find differences in fascicle lengths between compartments. However, pennation angles were on average $12^{\circ} \operatorname{larger}(p<0.01)$ in the posterior compartments than in the anterior compartments. For every centimetre that the muscle-tendon unit lengthened, fascicle lengths increased by $3.7 \pm 0.8 \mathrm{~mm}$, pennation angles decreased by $-3.2 \pm 0.9^{\circ}$ and curvatures decreased by $-2.7 \pm 0.8 / \mathrm{m}$. Fascicles in the posterior compartments rotated almost twice as much as in the anterior compartments during passive lengthening.

Discussion. The homogeneity in fascicle lengths and inhomogeneity in pennation angles of the soleus may indicate a functionally different role for the anterior and posterior compartments. The data and techniques presented here demonstrate how DTI can be used to obtain detailed, quantitative measurements of the anatomy of complex skeletal muscles in living humans. 


\section{Introduction}

42 The macroscopic arrangement of muscle fibres in the muscle belly is referred to as muscle architecture.

43 Muscle architecture is often quantified by parameters such as fascicle length, pennation angle and

44 physiological cross-sectional area (PCSA). Muscle architecture differs markedly between muscles and

45 individuals (Ward et al., 2009, Woittiez, 1984), and changes with age (Narici et al., 2008, Siebert et al.,

46 2017, Weide et al., 2015), exercise (Blazevich, 2006) and disease (Shortland et al., 2002, Foran et al.,

47 2005). To study these processes, quantitative methods to measure muscle- and subject-specific

48 architectural parameters are required. In this study, we use magnetic resonance imaging (MRI)

49 techniques to quantify the complex architecture of the human soleus muscle in vivo.

50 The human soleus has a complex, three-dimensional (3D) architecture. Studies of cadaver muscles using

51 micro-dissection techniques and magnetic resonance imaging have shown that the soleus is

52 compartmentalised: it consists of a unipennate posterior part wrapped around a radially bipennate

53 anterior part (Agur et al., 2003, Hodgson et al., 2006). The fascicles in each compartment have distinctly

54 different orientations but similar lengths (Agur et al., 2003). The large volume $\left(\sim 425 \mathrm{~cm}^{3}\right.$ in vivo (Lee et

55 al., 2006)) and short fascicle lengths (3-4 cm (Agur et al., 2003)) give the soleus the largest physiological

56 cross-sectional area of any human lower limb muscle (Ward et al., 2009).

57 The complexity of the soleus' architecture is also reflected in the connective tissues, examined in great

58 detail by Hodgson et al. (2006). Distally, the muscle is connected to the calcaneus through the rope-like

Achilles tendon, which the soleus shares with the gastrocnemius muscle. Just proximal to its insertion on

the calcaneus, the Achilles tendon has an elliptical cross-section in the transverse plane, but more

61

proximally it becomes wider and thinner as it joins the sheet-like posterior aponeurosis of the soleus

62 (Hodgson et al., 2006, Finni et al., 2003b, Balius et al., 2013). The distal ends of muscle fascicles in the posterior compartment of the soleus insert on the posterior aponeurosis and the proximal ends of the 
64

65

muscle fascicles originate from the posterior side of the anterior aponeurosis. The anterior aponeurosis (the origin of the muscle) forms another curved sheet of connective tissue that extends almost the entire length of the muscle belly, connecting the muscle proximally to the tibia and fibula and separating the muscle into posterior and anterior compartments. The anterior aspect of the anterior aponeurosis provides the origin of muscle fascicles in the anterior compartment. Fascicles in the anterior compartment insert on a protrusion of the posterior aponeurosis called the medial septum, which separates the muscle into medial and lateral compartments and presents as a clearly identifiable Tshaped structure on transverse MRI images (Hodgson et al., 2006). The structural partitioning of the human soleus is also evident in the 3D branching of nerves (Loh et al., 2003), indicating that compartments may have functionally different roles (English et al., 1993).

There have been few reports of quantitative measurements of the three-dimensional architecture of the soleus in vivo. Quantification of the 3D architecture of the soleus in vivo is difficult using conventional techniques such as ultrasound. With ultrasound, measurements from the deeper (anterior and proximal) compartment of the soleus muscle is difficult because image quality is often poor, although this depends on the system (Barber et al., 2017, Rana et al., 2013, Lai et al., 2015, Chow et al., 2000, Martin et al., 2001). Also, in the soleus it is difficult to orient the transducer in the plane of fascicles and perpendicular to the aponeurosis (Rana et al., 2013), as is required for accurate measurements of muscle architecture (Bolsterlee et al., 2016, Lee et al., 2015).

Three-dimensional imaging techniques such as magnetic resonance imaging (MRI) overcome some of the limitations of ultrasound (Finni et al., 2003a, Balius et al., 2013, Hodgson et al., 2006). MRI has been used to quantify lengths of the whole muscle belly and the structure of connective tissues, and to map intramuscular velocities during isometric contractions (Finni et al., 2003a, Hodgson et al., 2006). However, anatomical MRI scans lack the resolution to discern individual muscle fibres, which precludes 
87 measurement of key indices of muscle architecture such as fascicle lengths and pennation angles. For

88 this reason, there are limited data on fascicle lengths and pennation angles in the human soleus in vivo

89 (see Agur et al. (2003) for an overview of the available data). Also, it is largely unknown whether architectural parameters are uniform or differ between compartments. Non-uniformities could indicate as yet unrevealed functional differences between the soleus compartments.

Here, we use diffusion tensor imaging (DTI) to quantify the three-dimensional architecture of the human soleus muscle in vivo (Damon et al., 2017, Van Donkelaar et al., 1999, Bolsterlee et al., 2017, Oudeman et al., 2015). DTI is a magnetic resonance imaging (MRI) technique which exploits the principle that longitudinally arranged microstructures in muscles (such as cell membranes) hinder the diffusion of water molecules more in the plane perpendicular to the muscle fibre's long axis than along that axis. This principle can be used to measure fibre orientation (Damon et al., 2002) and - most interestingly for anatomical studies - to quantify the 3D muscle architecture using DTI fibre tractography algorithms (Damon et al., 2017, Yeh et al., 2013, Mori et al., 1999).

We have recently extended DTI tractography techniques by constraining the fibre tracts to terminate at the surface of the muscle (Bolsterlee et al., 2017). The muscle surface is located using MRI. Here, we apply these novel techniques that combine anatomical MRI with DTI data to quantify the complex architecture of the human soleus in vivo at two different muscle lengths. We provide the most detailed data to date of soleus muscle architecture, and changes in soleus muscle architecture with passive lengthening.

\section{Materials \& Methods}

107 We further analysed MRI and DTI data obtained from the left lower legs of six subjects (Table 1) of a 
109 procedures conformed to the Declaration of Helsinki (2008) and were approved by University of New

110 South Wales' Human Research Ethics Committee (HC15006; in vivo data) or Northern Sydney Local

111 Health District (RESP/16/9; cadaver data). Written informed consent of all subjects was obtained prior to

112 their participation.

113 Of the original eight participants in our previous study (Bolsterlee et al., 2017), data from one

114 participant were excluded because of poor image quality, thought to have been caused by movement

115 during scanning. Data from another participant were excluded because DTI fibre tractography generated

116 sparse fibre tracts (see section "Muscle architecture measurements").

\section{MRI and DTI scans}

118 For each participant, we analysed two sets of MRI and DTI scans obtained under passive conditions with

119 the ankle in a dorsiflexed position (condition 'long') and in a plantarflexed position (condition 'short').

An MRI-compatible frame was used to maintain the ankle in a fixed position. The frame could be locked

121 at different angles to reposition the ankle. For all scans, the participants lay supine with the knee slightly

122 flexed (Table 1). The ankle was passively dorsiflexed to an angle that corresponded to the slack length of

123 the medial gastrocnemius muscle, determined using ultrasound imaging (mean $\pm S D=69 \pm 12^{\circ}$, where

$12490^{\circ}$ means the sole of the foot is perpendicular to the tibia and values above $90^{\circ}$ indicate dorsiflexion;

125 see Bolsterlee et al. (2017) for details). The dorsiflexed position was the maximal dorsiflexion angle the

126 participant could comfortably achieve $\left(108 \pm 7^{\circ}\right)$. Thus, the ankle angles differed slightly between

127 participants (Table 1). The change in muscle-tendon length between the plantarflexed and dorsiflexed

128 position was estimated using regression equations (Grieve et al., 1978).

129 All scans were made using a 3T MRI scanner (Achieva 1.2, Philips Medical Systems, Best, The

130 Netherlands) with a 16-element SENSE XL torso coil. In each joint position, 75 transverse anatomical

131 images and DTI images were obtained covering the entire cross-section of the left lower leg from the 
132 proximal end of the tibia to the ankle (or for taller subjects, close to the ankle). The settings for the

133 anatomical scan were: TSE sequence, TR/TE 1842/8 ms, field of view (FOV) 180mm, acquisition matrix

$134288 \times 215$ (reconstructed to $960 \times 960$ ), voxel size $0.1875 \times 0.1875 \times 5 \mathrm{~mm}$ and scan time $320 \mathrm{~s}$. The settings

135 for the DTI scans were: DT-EPI sequence, TR/TE $8522 / 63 \mathrm{~ms}$, FOV $180 \mathrm{~mm}$, voxel size $1.875 \times 1.875 \times 5$

$136 \mathrm{~mm}, 16$ gradient directions on a hemisphere, number of signal averages $2, \mathrm{~b}=500 \mathrm{~s} / \mathrm{mm} 2$ (B0 image

137 with $\mathrm{b}=0 \mathrm{~s} / \mathrm{mm} 2$ ) and scan time $298 \mathrm{~s}$.

138 The DTI data were corrected for eddy current distortions (Andersson and Sotiropoulos, 2016) and

139 filtered with a local principal component analysis filter that smooths the raw DTI data while maintaining

140 sharp transitions between adjacent muscles (Manjon et al., 2013). Subsequently, the diffusion tensor

141 was reconstructed using DSI Studio (dsi-studio.labsolver.org; Yeh et al., 2013), from which the primary,

142 secondary and tertiary diffusion eigenvalues and eigenvectors were derived. A map of the primary

143 eigenvector (which indicates the primary diffusion direction, and thus the fibre orientation) was created.

\section{Muscle segmentation}

145 The soleus muscle was manually outlined on all of the slices of the T1-weighted anatomical scans that

146 the muscle was visible on (on average, 59 slices per scan with a spacing of $5 \mathrm{~mm}$ between slices) using 3D

147 Slicer (www.slicer.org - Fedorov et al., 2012). All segmentations were performed or checked by a

148 researcher (author TF) experienced in studying soleus anatomy (Finni et al., 2003a, Finni et al., 2003b,

149 Hodgson et al., 2006). It was apparent that the muscle was subdivided in four compartments (Fig. 1a):

150 medial-anterior (MA), lateral-anterior (LA), medial-posterior (MP) and lateral-posterior (LP), which is

151 consistent with previous anatomical descriptions (Agur et al., 2003, Finni et al., 2003a).

152 On most slices of the anatomical scan the boundaries of each compartment could clearly be identified,

153 but in the more proximal regions the boundaries within the muscle were sometimes unclear. To guide

154 and check the manual outlining, the segmentations made on the anatomical scans were overlayed on 
155 the primary eigenvector map derived from the DTI data using image processing software ITK-SNAP

156 (www.itksnap.org; Yushkevich et al., 2006). The primary eigenvector map contains a 3-component

157 direction vector indicating the 3D fibre direction in each voxel of the DTI scan, which can be visualised as

158 a colour-coded map where different colours indicate different fibre orientations (Fig. 1b). As different

159 muscle compartments have clearly different fibre orientations, the map assisted in drawing the

160 boundaries between compartments on slices where the boundaries were unclear on the anatomical

161 scan. For this procedure we used ITK-SNAP to overlay the primary eigenvector map on the anatomical

162 scan with interactively changeable transparency, facilitating optimal use of both anatomical and DTI

163 data sources for manual segmentation. As an indicator of reliability of the segmentation, we calculated

164 root mean squared differences in muscle volume between scans obtained at short and long muscle

165 lengths. As muscle volume is likely to change less than $2 \%$ with passive lengthening (Bolsterlee et al.,

166 2017), larger differences in volume between muscle lengths presumably indicate errors in segmentation.

\section{Muscle architecture measurements}

168 From the segmentations, 3D triangulated surface models were created for all compartments using the

169 MATLAB-based iso2mesh toolbox (Fig. 1c and 1e; Fang and Boas, 2009). Muscle volume of each

170 compartment was calculated as the volume of the surface models. Muscle length was calculated as the

171 distance between the most proximal and the most distal point of the surface models of all

172 compartments combined, projected along the long axis of the muscle. The long axis of the muscle was

173 approximated by a line connecting a proximal and distal point on the anterior surface of the soleus

174 between the medial-anterior and lateral-anterior compartment (Fig. 1c-d)

175 Deterministic DTI fibre tracking algorithms built into DSI Studio (Yeh et al., 2013) were used to generate

1761,000 fibres in each muscle compartment. Fibre tracking is the procedure of generating curves that,

177 starting from a seed point, follow the primary direction of diffusion bi-directionally through a DTI scan 
178 volume (Mori et al., 1999). When performed in muscle tissue, these curves follow the fibre orientation

179 throughout a muscle (Damon et al., 2002) and, when appropriate stopping criteria are defined

180 (Heemskerk et al., 2009), they resemble muscle fibres (Damon et al., 2017). We used randomly placed

181 seed points located inside the muscle but at least two voxels away from the muscle surface. Fibre

182 tracking is often inaccurate at the boundary of the muscle (Sinha et al., 2015), possibly because of

183 erroneous estimation of the fibre direction in voxels that contain signal from more than one muscle

184 (Oudeman et al., 2016). Therefore, we terminated fibre tracking when the tract entered the boundary

185 layer of a muscle. The seed and boundary regions were created by resampling the masks created on the 186 anatomical scans to the resolution of the DTI scans. We used fibre tracking settings similar to those used 187 previously by our group and others : $0.1 \leq$ fractional anisotropy $\leq 0.5,1 \cdot 10^{-3} \mathrm{~mm}^{2} / \mathrm{s} \leq \mathrm{mean}$ diffusivity $\leq$ $1882 \cdot 10^{-3} \mathrm{~mm}^{2} / \mathrm{s}$, maximum angle between subsequent tract segments $=10^{\circ}, 15 \mathrm{~mm} \leq$ fibre tract length $\leq$ $189200 \mathrm{~mm}$, step size $=1 \mathrm{~mm}$. Seeding was continued until 1,000 tracts satisfying these constraints were 190 found.

191 Fibre tracts frequently do not terminate on aponeuroses or tendons, and thus the ends of fibre tracts do

192

193 not represent the ends of muscle fascicles. To find origin and insertion points for each fibre tract we used algorithms to ensure that fibre tracts attach to aponeuroses (or, more precisely, to 3D surface models generated from the manually outlined muscles) (Bolsterlee et al., 2017). Briefly, the 3D surface models from the anatomical scans were overlayed on the fibre tracts and a third-order polynomial curve was fitted in $3 \mathrm{D}$ to the fibre tracts. The polynomial curve was linearly extended at both end points using the slope of the curve at the end point until the extension intersected the muscle surface. The polynomial curves fitted on the raw fibre tracts, including their extrapolations to the muscle surface, will be referred to as fascicles. The extrapolation procedure reduces the variability of fascicle lengths within a muscle and presumably makes the results less sensitive to the fibre tracking settings than if tracts are not extrapolated (Bolsterlee et al., 2017). 
202 Fascicle lengths were calculated as the sum of the length of the polynomials curves (fitted on the raw

203 fibre tracts) and the extrapolations. We also calculated fascicle lengths as a fraction of whole-muscle

204 length. Pennation angles were calculated in 3D as the angle between the line connecting the origin and

205 insertion of a fascicle and the long axis of the muscle (Fig. 1c-d). Using the Frenet-Serret formula, the

206 curvature of a fascicle (expressed as $1 /$ radius $\left[\mathrm{m}^{-1}\right]$ ) was calculated as the mean curvature of 100

207 equidistant points along the polynomial curve of that fascicle.

208 Of the 1,000 fascicles that were reconstructed in each compartment, only fascicles extended by less

209 than $50 \%$ of their total length and less than $20 \mathrm{~mm}$ were included in further analyses. All fascicles were

210 visually inspected for plausibility and to verify the distribution of fascicles throughout each

211 compartment (Fig. 1d and 1f). Mean muscle architecture measurements for a compartment were

212 calculated by averaging architecture measurements from all successfully reconstructed fascicles in that

213 compartment. Within each compartment, physiological cross-sectional area (PCSA) was calculated by

214 dividing muscle volume by fascicle length.

\section{Statistics}

216 We used linear mixed models to investigate differences between compartments in architecture (mean

217 fascicle length, pennation angle and curvature) at the short muscle length. In these models, subjects

218 were assigned random intercepts and there were fixed effects for muscle compartment.

219 We also used linear mixed models to quantify changes in architecture with passive lengthening. These

220 models estimated the change in architecture per centimetre change in muscle-length, and tested for

221 differences between compartments in change in architecture with passive lengthening. Subjects were

222 assigned random intercepts and there were fixed effects for muscle compartment, muscle length and

223 the interaction between muscle compartment and muscle length. 


\section{Cadaver measurements}

225 To confirm the soleus anatomy as reconstructed with DTI, we measured the architecture of two

226 cadaveric human soleus muscles using 3D microdissection techniques similar to the techniques used by

227 Lee et al. (2015). The muscles were harvested from the right legs of fresh-frozen cadavers of a 67-year

228 old male (muscle 1; muscle length: $36.5 \mathrm{~cm}$ ) and a 90-year old male (muscle 2; muscle length: $32 \mathrm{~cm}$ ).

229 After gross dissection, the soleus muscles were fixed by submerging the tissue in a $10 \%$ neutral buffered

230 formalin solution for 11 weeks. The architecture of the fixed soleus was reconstructed using a 3D spatial

231 digitiser (MicroScribe G2X, Immersion Corp., San Jose, USA), connected to a laptop computer. The tip of

232 the digitiser was run from origin to insertion of a clearly visible fascicle bundle while recording the 3D

233 coordinates of the tip with a sampling frequency of $20 \mathrm{~Hz}$ (leading to $1 \mathrm{~mm}$ spacing between points; Fig.

234 2). After digitisation, the fascicle bundle was removed carefully using scalpel and tweezers, exposing the

235 deeper layers of fascicles which were then digitised and removed. This procedure was repeated until no

236 muscle tissue was left. We digitised only those fascicles of which the entire course from origin to

237 insertion could clearly be identified. To limit movement of the muscle relative to the base of the digitiser

238 (i.e. relative to the fixed coordinate system of the digitiser), the muscle was cast in a custom-made

239 silicon mould during dissection and digitisation.

240 Fascicle length was calculated as the sum of the lengths of the line segments connecting the points

241 along the course of one fascicle. Pennation angle was calculated in 3D as the angle between the line

242 connecting the first and last point on a fascicle, and the line connecting two points on the anterior

243 surface of the medial septum (considered an approximation of the line of action of the soleus). Mean

244 fascicle lengths and pennation angles were calculated for all four compartments of both muscles.

245 We did not control for ankle angle during gross dissection of the soleus muscle, and muscle length was

246 not fixed during fixation, so we do not know the ankle angle corresponding to the architecture 
247 measurements reported for the dissections. During fixation the muscles were hung vertically from their

248 proximal ends in a cylindrical tube with a small weight $(\sim 0.5 \mathrm{~kg})$ attached to the distal end, so the

249 tension in the tissue was small. Therefore, the muscle length is likely to correspond to a more

250 plantarflexed ankle position, which is comparable to the 'short' condition in the in vivo data.

\section{Results}

252 Four distinct compartments were apparent on the MRI and DTI scans of all six participants (Fig. 1a). The posterior compartment had, on average, four times the volume of the anterior part, although there was some inter-individual variation (Fig. 3, Table 2). The average total muscle volume was $356 \pm 58 \mathrm{~cm}^{3}$. On average, muscle volumes in all compartments were similar between measurements made at short and long muscle lengths (Table 2). The root mean squared difference between compartment volumes measured at short and long muscle lengths was $5.7 \mathrm{~cm}^{3}$ or $6.3 \%$ of the average compartment volume. The root mean squared difference between total muscle volumes (sum of four compartments) measured at short and long lengths was $14.1 \mathrm{~cm}^{3}$ or $3.9 \%$ of the average muscle volume.

From the 1,000 fibres tracked in each muscle compartment, on average, 600 fascicles were successfully $\pm 1.3 \mathrm{~mm}(29.4 \pm 4.5 \%$ of the fascicle length). In all compartments fascicles were reconstructed throughout most of the muscle belly (Fig. 1C-f), although fewer fascicles were successfully reconstructed in the most proximal and distal ends of the soleus. The DTI-based muscle fascicle reconstructions confirmed the presence of a bipennate anterior compartment and a unipennate posterior compartment 266 in all subjects (Fig. 1). 
269 respectively (Fig. 4, Table 2). Average fascicle lengths in the soleus ranged from 29 to $51 \mathrm{~mm}$ between

270 individuals (Fig. 5). As a proportion of muscle length, average fascicle lengths were $0.13 \pm 0.03$. Within

271 individuals we did not find statistically significant differences between compartments in fascicle lengths

272 (Fig. 5a). Pennation angles, however, were significantly larger in the posterior compartments than in the

273 anterior compartments by, on average, $12^{\circ}$ (Fig. 5b). Also, curvatures were $4 / \mathrm{m}$ larger in the posterior

274 compartments compared to the anterior compartments (Fig. 5c). We did not detect statistically

275 significant differences in architecture between lateral and medial compartments.

276 Passive lengthening of the soleus muscle-tendon unit by $30 \pm 8 \mathrm{~mm}$ resulted in changes in architecture

277 that were consistent between subjects (Fig. 6). For every centimetre that the muscle-tendon unit

278 lengthened, fascicle lengths increased by $3.7 \pm 0.8 \mathrm{~mm}$, pennation angles decreased by $3.2 \pm 0.9^{\circ}$ and

279 curvatures decreased by $2.7 \pm 0.8 / \mathrm{m}$. Although the fascicle length change was significantly different

280 between the medial-anterior and lateral-anterior compartment, we generally found small and non-

281 significant differences between medial and lateral compartments in changes in architecture. Pennation

282 angles decreased more in posterior compartments $\left(-4.2 \pm 1.1^{\circ} / \mathrm{cm}\right.$ muscle-tendon lengthening $)$ than in

283 anterior compartments $\left(-2.3 \pm 0.8^{\circ} / \mathrm{cm}\right.$; Fig. $\left.6 \mathrm{~b}\right)$.

284 Cadaver dissections confirmed the four-compartment architecture of the soleus in both cadaveric

285 muscles. Lengths and pennation angles of 994 fascicles were measured in two muscles with a minimum

286 of 58 fascicles per compartment (Table 3). Dissection measurements of fascicle lengths were similar to

287 those measured using DTI. Dissection measurements of anterior compartment pennation angles were

288 similar to those measured with DTI, but dissection measurements of posterior compartment pennation

289 angles were smaller than those measured by DTI. In contrast to measurements made with DTI,

290 dissection measurements of pennation in the posterior and anterior compartments were similar. 


\section{Discussion}

292 This study used novel DTI-based techniques to discern the complex 3D anatomy of the human soleus

293 muscle in vivo. The data confirmed the four-compartment structure of the soleus previously described in

294 cadaver muscles. There was evidence of differences in pennation angles and curvature, but not fascicle

295 lengths, between the posterior and anterior compartments. Importantly, this is the first study to report

296 inter-compartment differences in change in architecture of the human soleus with passive lengthening.

297 The gross anatomical data we present here are largely comparable with previous studies with four

298 identified compartments (Agur et al., 2003, Hodgson et al., 2006). However, we did not observe, with

299 either the imaging procedures or the dissection, a fifth marginal soleus compartment, as described by

300 Agur et al. (2003). The marginal soleus was also not discerned in the Visible Human Dataset (Sinha et al., 301 2011).

302 Fascicle lengths and pennation angles fall within the range previously reported on cadaver muscles

303 (Agur et al., 2003, Ward et al., 2009, Spoor et al., 1991) and in vivo using ultrasound (Stenroth et al., 304 2016, Kawakami et al., 1998, Maganaris, 2001, Maganaris et al., 1998). Although fascicle lengths

305 between compartments were similar, there was considerable variation in average fascicle lengths

306 between subjects, ranging from 29 to $51 \mathrm{~mm}$ at the short muscle length and 37 to $61 \mathrm{~mm}$ at the long

307 length. Soleus muscle volumes in our dataset (mean $356 \mathrm{~cm}^{3}$ ) were larger than those measured in

308 cadaver muscles (260 cm³; Ward et al. (2009)), but smaller than previously measured in vivo (489 - 550

$309 \mathrm{~cm}^{3}$; Hodgson et al. (2006), (Fukunaga et al., 1992)). Sarcopenia in the muscles of the elderly cohort

310 studied by Ward et al. likely explains the smaller soleus volumes in their sample. The predominantly

311 male participants ( 11 male, 1 female) studied by Fukunaga et al. were, on average, $5 \%$ taller and $17 \%$

312 heavier than our participants ( 3 male, 3 female), explaining at least partially why the soleus in

313 Fukunaga's sample was larger. Hodgson et al. did not report the gender, height or weight of the three 
314 participants for which they reported muscle volumes. In our six participants, the posterior

315 compartments were 2.7 to 8.9 times larger than the anterior compartments, confirming previous

316 reports of large inter-individual variations in relative volume between compartments (Hodgson et al.,

317 2006). The larger PCSA and smaller pennation angles of the posterior compartments indicate a muscle

318 design more geared towards high force production. The smaller PCSA and pennation angles of the

319 anterior compartment may indicate a role more geared towards establishing muscle excursion, rather

320 than force production. However, we did not detect differences between compartments in fascicle

321 length/muscle length ratio, which indicates whether the design of a muscle is more geared towards

322 providing large excursions (high values) or high forces (low values). The low ratio $(0.13 \pm 0.3)$ is line with

323 previous cadaver measurements (0.11; Ward et al., 2009) and confirms the role of the soleus in

324 generating high forces over a small range of lengths.

325 This study is the first to report inter-compartment differences in change in architecture of the human

326 soleus with passive lengthening. The medial anterior compartment showed the most pronounced

327 change in length of $14 \mathrm{~mm}$ that was significantly different from that in the lateral anterior compartment. We observed larger fascicle rotations in the posterior compartments, which could be indicative of a functionally different role of the posterior and anterior compartments. Functional partitioning of the soleus was suggested when it was found that nerve branching in the human soleus follows the structural compartmentalisation of the muscle (Loh et al., 2003). This provides a possible mechanism for independent control of different compartments. A new observation contributed by the current study is that this compartmentalisation is also reflected in differences in passive mechanical properties of the compartments. To the extent that the observed inter-compartmental differences are 
337 Few other studies have reported changes in 3D muscle architecture in the soleus with passive

338 lengthening. Sinha et al. (2011) also used DTI techniques and reported changes in fascicle orientation

339 (or, more precisely, changes in the primary eigenvector of the diffusion tensor) up to $46^{\circ}$ with $30^{\circ}$ ankle

340 rotation - much larger than the changes we report here $\left(9.4^{\circ}\right.$ change in pennation over $39^{\circ}$ ankle

341 rotation). Whereas Sinha and colleagues reported changes in the primary eigenvector relative to the

342 long axis of the scanner, we measure pennation angles from fascicles reconstructions relative to the

343 long axis of the muscle. The fascicle reconstructions were bound by anatomical constraints, and

344 implausible fascicles were excluded, presumably making our measurement more accurate. Rana et al.

345 (2013) used 3D ultrasound techniques to measure changes in fibre orientation (but not fascicle lengths)

346 with plantarflexion contractions and ankle rotation. They reported only $0.8^{\circ}$ rotation of fascicles in the

347 soleus (relative to the muscle's long axis) with $45^{\circ}$ ankle rotation, which is surprisingly small compared

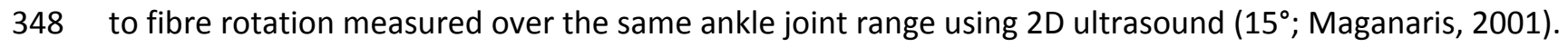

349 The change in pennation angles and fascicle lengths we report here are much closer to 2D ultrasound-

350 measured values from the posterior compartment (Maganaris, 2001), and physiologically more

351 plausible. Note that the values we report represent a linear (i.e. average) estimate of the possibly non-

352 linear effect of muscle-tendon lengthening on changes in muscle architecture (Herbert et al., 2011).

353 The DTI-based reconstruction techniques used here improve on conventional DTI fibre tracking

354 algorithms. Tractography algorithms generate curves that follow the direction in which most diffusion

355 occurs (i.e. the muscle fibre direction) throughout a muscle. The lengths of these curves are often

356 interpreted as muscle fibre lengths, and the orientation of the curves is often used to calculate

357 pennation angles (Sinha et al., 2011, Oudeman et al., 2016). Unfortunately, the fibre tracts are only

358 moderately reproducible (Heemskerk et al., 2009). Application of this approach to measuring fascicle

359 lengths in the soleus has resulted in measurement of fibre lengths ranging from $\sim 1-2 \mathrm{~cm}$ (Sinha et al.,

360 2011) to 5-6 cm (Oudeman et al., 2016). These values depart substantially from the findings of the most 
361 detailed dissection study, which reported fascicle lengths of 3-4 cm (Agur et al., 2003), and other

362 cadaver studies that report similar measurements (Ward et al., 2009, Spoor et al., 1991).

363 In the present study we used information from anatomical scans about the location of the aponeuroses

364 to force fibre tracts to terminate on tendons or aponeuroses. In our opinion, these constraints are

365 necessary to obtain realistic measurements of muscle architecture. We also expect that constraining

366 fibre tracts to end on aponeuroses reduces the sensitivity of the architecture measurements to

367 variations in fibre tracking settings; however, this hypothesis requires formal testing. Although direct

368 validation was not possible, the consistent and physiologically plausible changes in architecture we

369 observed in all muscle compartments in all participants provide some evidence for the validity of our

370 techniques. Extrapolation of fibre tracts to aponeuroses requires segmentation of muscles from scans,

371 which is time-consuming and prone to error. We found that manual segmentation was aided greatly by

372 overlaying the primary eigenvector map on the anatomical scan (as detailed in the Methods section) to

373 make optimal use of information about muscle boundaries from the two data sources. The root mean

374 squared difference in volumes of the soleus' compartments between short and long lengths was $6.3 \%$,

375 indicating a reasonable reliability of manual segmentation in this complex muscle. Some, but probably

376 not all, of this difference may be attributed to actual changes in volume with passive stretching, as we

377 showed earlier in this dataset that medial gastrocnemius muscle volume reduced by $1.6 \%$ from short to

378 long muscle lengths (Bolsterlee et al., 2017). We did not determine the extent to which inaccuracies in

379 manual muscle segmentations have propagated to errors in muscle architecture measurements. Future

380 development of (semi-)automatic muscle segmentation algorithms will be of great benefit to obtain

381 accurate, reproducible measurements of muscle architecture from DTI. A possible pathway to

382 development of such algorithms is to use information from both anatomical and DTI scans, rather than

383 only from anatomical scans (e.g. Gilles and Magnenat-Thalmann, 2010). 
384 The DTI-based measurements of muscle architecture were compared to measurements made using

385 microdissection techniques on two cadaveric muscles. Similar measurements of fascicle lengths and

386 pennation angles in the anterior compartments were obtained with the two approaches. However, the

387 dissection did not reproduce the finding made with DTI that pennation angles in the posterior

388 compartments are larger than in the anterior compartments. This inconsistency between the dissection

389 and DTI data might be explained by an altered orientation of the posterior compartments relative to the

390 long axis of the muscle caused by removing the muscle from the surrounding anatomical structures (e.g.

391 the gastrocnemius). It is also possible that age-related changes affect the pennation angles of the

392 posterior compartments differently compared to the anterior compartments.

Unfortunately, we were not able to obtain high-quality DTI measurements from the same muscles that

we dissected. Prior to dissection we scanned the cadaver muscles using the same DTI scanning protocol

as used in vivo, and we applied the same algorithms for reconstruction, but the quality of the

reconstructions was poor. It is likely that freeze-thawing or fixing the muscle altered the diffusion

properties of the tissue. It is difficult or impossible to procure whole human muscles that have not been

freeze-thawed or fixed. This precludes direct validation of DTI measurements of muscle architecture on

human muscles. For now, direct validation of DTI-based measurement of muscle architecture are best

carried out by comparing DTI and dissection measures on animal muscles (Schenk et al., 2013, Damon et

al., 2002), or with phantoms that resemble muscle tissue (Berry et al., 2017).

402

403

404

405

406

Classical anatomical textbooks provide qualitative descriptions and schematic images of muscle architecture which are valuable for gaining a general understanding of musculoskeletal function. But more detailed quantitative analyses, for instance with muscle models, require quantitative data on muscle architecture. All of the existing datasets of muscle architecture have been obtained from cadaver muscles, often obtained from the cadavers of elderly people at least some of whom have sarcopenia. 
407 The techniques we present here will be useful in the development of three-dimensional, quantitative

408 atlases of human muscle architecture in vivo.

409

410

411

412

413

414

415

416

417

418

419

420

421

422

423

424

425

426

427

428

429

430

431

432

433

434

435

436

437

438

439

\section{Conclusions}

Using a novel DTI-based method this study presented comprehensive data of the 3D architecture of the

four compartments of human soleus muscle in vivo. Importantly, the results provided a first look on 3D

changes in the compartmentalised soleus muscle during passive lengthening that may provide reference

values for future muscle models. The DTI techniques that we applied here to quantify the architecture of the soleus could be used to develop comprehensive, quantitative atlases of human muscle architecture.

\section{References}

Agur AM, Ng-Thow-Hing V, Ball KA, Fiume E, McKee NH (2003) Documentation and three-dimensional modelling of human soleus muscle architecture. Clin Anat, 16, 285-293.

Andersson JLR, Sotiropoulos SN (2016) An integrated approach to correction for off-resonance effects and subject movement in diffusion MR imaging. Neuroimage, 125, 1063-1078.

Balius R, Alomar X, Rodas G, Miguel-Perez M, Pedret C, Dobado MC, Blasi J, Koulouris G (2013) The soleus muscle: $\mathrm{MRI}$, anatomic and histologic findings in cadavers with clinical correlation of strain injury distribution. Skeletal Radiol, 42, 521-530.

Barber L, Carty C, Modenese L, Walsh J, Boyd R, Lichtwark G (2017) Medial gastrocnemius and soleus muscle-tendon unit, fascicle, and tendon interaction during walking in children with cerebral palsy. Dev Med Child Neurol, 59, 843-851.

Berry DB, You ST, Warner J, Frank LR, Chen SC, Ward SR (2017) A 3D tissue-printing approach for validation of diffusion tensor imaging in skeletal muscle. Tissue Eng Pt A, 23, 980-988.

Blazevich AJ (2006) Effects of physical training and detraining, immobilisation, growth and aging on human fascicle geometry. Sports Med, 36, 1003-1017.

Bolsterlee B, D'Souza A, Gandevia SC, Herbert RD (2017) How does passive lengthening change the architecture of the human medial gastrocnemius muscle? J Appl Physiol, 122, 727-738.

Bolsterlee B, Gandevia SC, Herbert RD (2016) Effect of transducer orientation on errors in ultrasound image-based measurements of human medial gastrocnemius muscle fascicle length and pennation. PLOS One, 11.

Chow RS, Medri MK, Martin DC, Leekam RN, Agur AM, McKee NH (2000) Sonographic studies of human soleus and gastrocnemius muscle architecture: gender variability. Eur J Appl Physiol, 82, 236244.

Damon BM, Ding ZH, Anderson AW, Freyer AS, Gore JC (2002) Validation of diffusion tensor MRI-based muscle fiber tracking. Magnet Reson Med, 48, 97-104. 
Damon BM, Froeling M, Buck AK, Oudeman J, Ding Z, Nederveen AJ, Bush EC, Strijkers GJ (2017) Skeletal muscle diffusion tensor-MRI fiber tracking: rationale, data acquisition and analysis methods, applications and future directions. NMR Biomed, $\mathbf{3 0}$.

English AW, Wolf SL, Segal RL (1993) Compartmentalization of muscles and their motor nuclei - the partitioning hypothesis. Physical Therapy, 73, 857-867.

Fang QQ, Boas DA (2009) Tetrahedral mesh generation from volumetric binary and gray-scale images. Proceedings of IEEE International Symposium on Biomedical Imaging, 1142-1145.

Fedorov A, Beichel R, Kalpathy-Cramer J, Finet J, Fillion-Robin J-C, Pujol S, Bauer C, Jennings D, Fennessy F, Sonka M (2012) 3D Slicer as an image computing platform for the Quantitative Imaging Network. Magn Reson Imaging, 30, 1323-1341.

Finni T, Hodgson JA, Lai AM, Edgerton VR, Sinha S (2003a) Mapping of movement in the isometrically contracting human soleus muscle reveals details of its structural and functional complexity. $J$ Appl Physiol, 95, 2128-2133.

Finni T, Hodgson JA, Lai AM, Edgerton VR, Sinha S (2003b) Nonuniform strain of human soleus aponeurosis-tendon complex during submaximal voluntary contractions in vivo. J Appl Physiol, 95, 829-837.

Foran JRH, Steinman S, Barash I, Chambers HG, Lieber RL (2005) Structural and mechanical alterations in spastic skeletal muscle. Dev Med Child Neurol, 47, 713-717.

Fukunaga T, Roy RR, Shellock FG, Hodgson JA, Day MK, Lee PL, Kwong-Fu H, Edgerton VR (1992) Physiological cross-sectional area of human leg muscles based on magnetic resonance imaging. $J$ Orthop Res, 10, 928-34.

Gilles B, Magnenat-Thalmann N (2010) Musculoskeletal MRI segmentation using multi-resolution simplex meshes with medial representations. Med Image Anal, 14, 291-302.

Grieve DW, Pheasant S, Cavanagh PR (1978) Prediction of gastrocnemius length from knee and ankle joint posture. In Biomechanics VI: Proceedings of the Sixth International Congress of Biomechanics), pp. 405-412. Copenhagen, Denmark: University Park Press Baltimore.

Heemskerk AM, Sinha TK, Wilson KJ, Ding ZH, Damon BM (2009) Quantitative assessment of DTI-based muscle fiber tracking and optimal tracking parameters. Magnet Reson Med, 61, 467-472.

Herbert RD, Clarke J, Kwah LK, Diong J, Martin J, Clarke EC, Bilston LE, Gandevia SC (2011) In vivo passive mechanical behaviour of muscle fascicles and tendons in human gastrocnemius muscletendon units. J Physiol, 589, 5257-5267.

Hodgson JA, Finni T, Lai AM, Edgerton VR, Sinha S (2006) Influence of structure on the tissue dynamics of the human soleus muscle observed in MRI studies during isometric contractions. J Morphol, 267, 584-601.

Kawakami Y, Ichinose Y, Fukunaga T (1998) Architectural and functional features of human triceps surae muscles during contraction. J Appl Physiol, 85, 398-404.

Lai A, Lichtwark GA, Schache AG, Lin YC, Brown NAT, Pandy MG (2015) In vivo behavior of the human soleus muscle with increasing walking and running speeds. J Appl Physiol, 118, 1266-1275.

Lee D, Li Z, Sohail QZ, Jackson K, Fiume E, Agur A (2015) A three-dimensional approach to pennation angle estimation for human skeletal muscle. Comput Methods Biomech Biomed Eng, 18, 14741484.

Lee HD, Finni T, Hodgson JA, Lai AM, Edgerton VR, Sinha S (2006) Soleus aponeurosis strain distribution following chronic unloading in humans: an in vivo MR phase-contrast study. J Appl Physiol, 100, 2004-2011.

Loh EY, Agur AM, McKee NH (2003) Intramuscular innervation of the human soleus muscle: A 3D model. Clin Anat, 16, 378-382.

Maganaris CN (2001) Force-length characteristics of in vivo human skeletal muscle. Acta Physiol Scand, $172,279-285$. 
Maganaris CN, Baltzopoulos V, Sargeant AJ (1998) In vivo measurements of the triceps surae complex architecture in man: implications for muscle function. J Physiol, 512, 603-614.

Manjon JV, Coupe P, Concha L, Buades A, Collins DL, Robles M (2013) Diffusion weighted image denoising using overcomplete local PCA. PLOS One, 8.

Martin DC, Medri MK, Chow RS, Oxorn V, Leekam RN, Agur AM, McKee NH (2001) Comparing human skeletal muscle architectural parameters of cadavers with in vivo ultrasonographic measurements. J Anatomy, 199, 429-434.

Mori S, Crain BJ, Chacko VP, van Zijl PCM (1999) Three-dimensional tracking of axonal projections in the brain by magnetic resonance imaging. Ann Neurol, 45, 265-269.

Narici MV, Maffulli N, Maganaris CN (2008) Ageing of human muscles and tendons. Disabil Rehabil, 30, 1548-1554.

Oudeman J, Mazzoli V, Marra MA, Nicolay K, Maas M, Verdonschot N, Sprengers AM, Nederveen AJ, Strijkers GJ, Froeling M (2016) A novel diffusion-tensor MRI approach for skeletal muscle fascicle length measurements. Physiological Reports, 4.

Oudeman J, Nederveen AJ, Strijkers GJ, Maas M, Luijten PR, Froeling M (2015) Techniques and applications of skeletal muscle diffusion tensor imaging: A review. J Magn Reson Imaging, 773788.

Rana M, Hamarneh G, Wakeling JM (2013) 3D fascicle orientations in triceps surae. J Appl Physiol, 115, 116-125.

Schenk P, Siebert T, Hiepe P, Gullmar D, Reichenbach JR, Wick C, Blickhan R, Bol M (2013) Determination of three-dimensional muscle architectures: validation of the DTI-based fiber tractography method by manual digitization. J Anatomy, 223, 61-68.

Shortland AP, Harris CA, Gough M, Robinson RO (2002) Architecture of the medial gastrocnemius in children with spastic diplegia. Dev Med Child Neurol, 44, 158-163.

Siebert T, Tomalka A, Stutzig N, Leichsenring K, Böl M (2017) Changes in three-dimensional muscle structure of rabbit gastrocnemius, flexor digitorum longus, and tibialis anterior during growth. $J$ Mech Behav Biomed Mater, 74, 507-519.

Sinha U, Csapo R, Malis V, Xue Y, Sinha S (2015) Age-related differences in diffusion tensor indices and fiber architecture in the medial and lateral gastrocnemius. J Magn Reson Imaging, 41, 941-953.

Sinha U, Sinha S, Hodgson JA, Edgerton RV (2011) Human soleus muscle architecture at different ankle joint angles from magnetic resonance diffusion tensor imaging. J Appl Physiol, 110, 807-819.

Spoor CW, Van Leeuwen JL, Van der Meulen WJTM, Huson A (1991) Active force-length relationship of human lower-leg muscles estimated from morphological data - a comparison of geometric muscle models. Eur J Morphology, 29, 137-160.

Stenroth L, Cronin NJ, Peltonen J, Korhonen MT, Sipila S, Finni T (2016) Triceps surae muscle-tendon properties in older endurance- and sprint-trained athletes. J Appl Physiol, 120, 63-69.

Van Donkelaar CC, Kretzers LG, Bovendeerd PHM, Lataster LMA, Nicolay K, Janssen JD, Drost MR (1999) Diffusion tensor imaging in biomechanical studies of skeletal muscle function. J Anatomy, 194, 79-88.

Ward SR, Eng CM, Smallwood LH, Lieber RL (2009) Are current measurements of lower extremity muscle architecture accurate? Clin Orth Rel Res, 467, 1074-1082.

Weide G, Huijing PA, Maas JC, Becher JG, Harlaar J, Jaspers RT (2015) Medial gastrocnemius muscle growth during adolescence is mediated by increased fascicle diameter rather than by longitudinal fascicle growth. J Anatomy, 226, 530-541.

Woittiez R (1984) A quantitative study of muscle architecture and muscle function. Amsterdam, The Netherlands: Vrije Universiteit Amsterdam.

Yeh FC, Verstynen TD, Wang YB, Fernandez-Miranda JC, Tseng WYI (2013) Deterministic diffusion fiber tracking improved by quantitative anisotropy. PLOS One, 8. 
536 Yushkevich PA, Piven J, Hazlett HC, Smith RG, Ho S, Gee JC, Gerig G (2006) User-guided 3D active 


\section{Figure 1}

Reconstruction of the architecture of the human soleus muscle using MRI and DTI.

(A) Transverse T1-weighted MRI slice approximately midway between the ankle and the knee. The four compartments of the soleus are outlined (blue = medial-posterior (MP), cyan $=$ lateral-posterior $(\mathrm{LP})$, red = medial-anterior $(\mathrm{MA})$, yellow = lateral-anterior $(\mathrm{LA})$ )

Corresponding slice of the primary eigenvector map from the DTI data (different colours indicate different diffusion directions (i.e. muscle fibre directions). The outlines of the soleus compartments from the anatomical scan are overlayed to show that the four compartments have different fibre orientations. (C) Anterior view of the left lower leg (top is superior) showing the 3D reconstruction of the surface of all muscle compartments based on the outlines on the anatomical scan. The skin is shown as a transparent overlay. (D) Anterior view of 3D fascicle reconstructions (after extrapolations of fibre tract end points to the muscle surface) of the same muscle as in (C). Fascicles are coloured per compartment. The dashed line in (C) and (D) represent the long axis of the muscle relative to which pennation angles were calculated. (E) Posterior view of the left lower leg showing the posterior compartments of the soleus and the skin (transparent). (F) Posterior view of 3D fascicle reconstructions. 
A

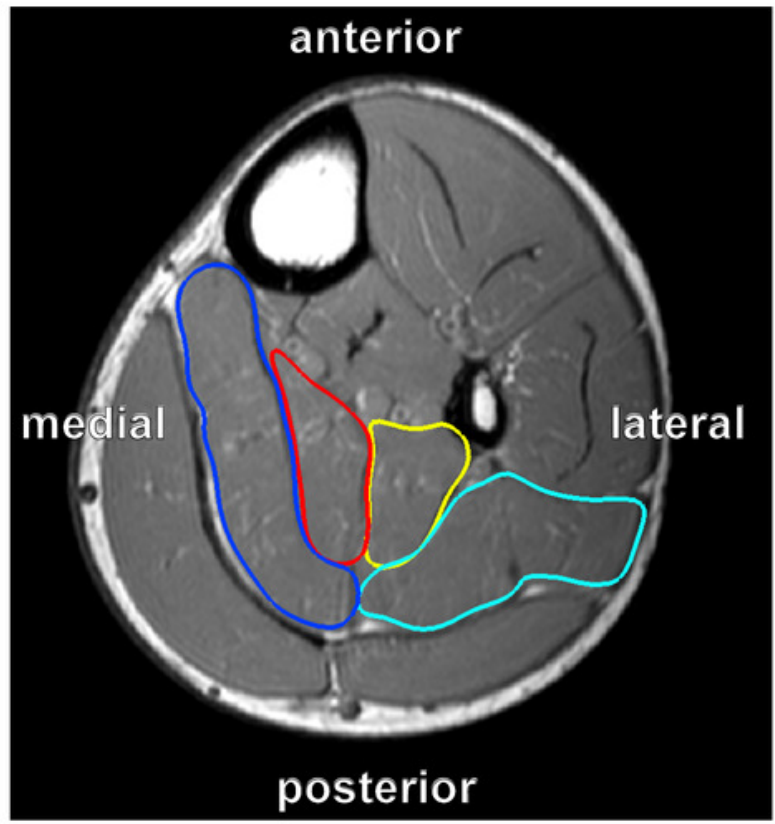

C

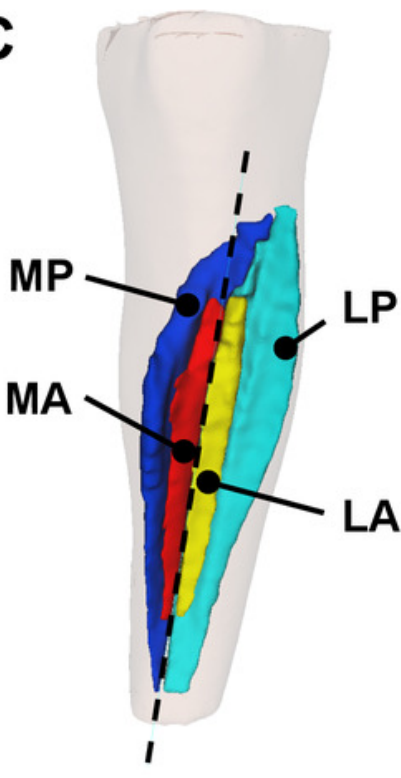

B

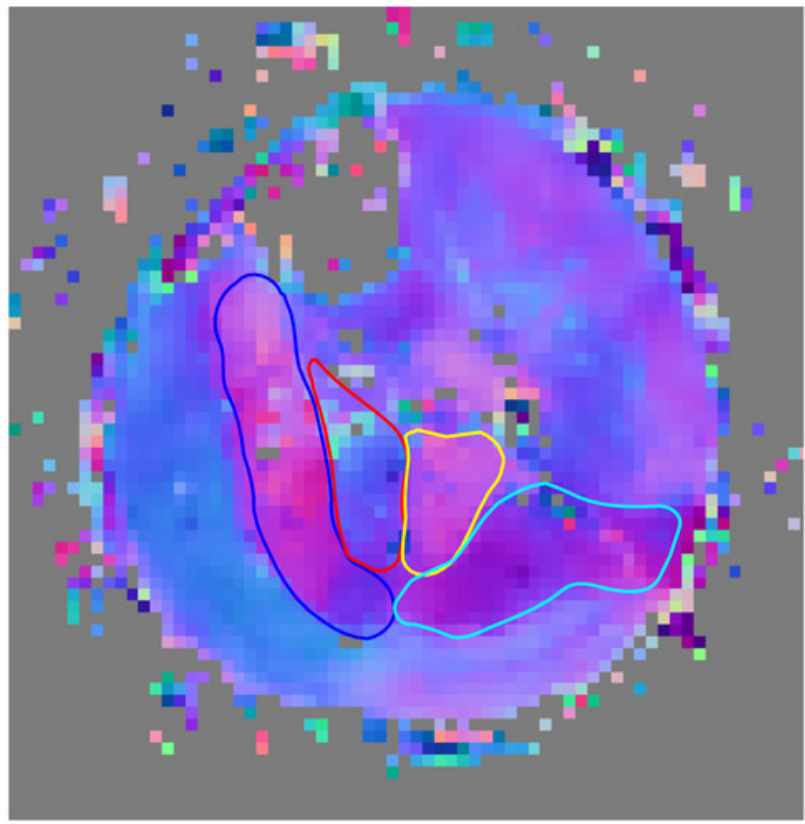

E

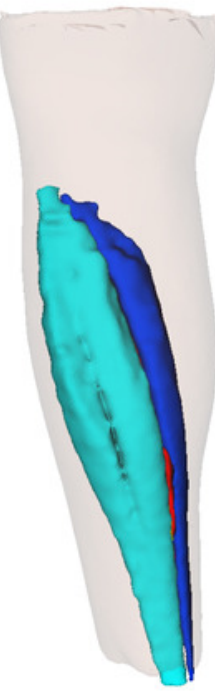

F

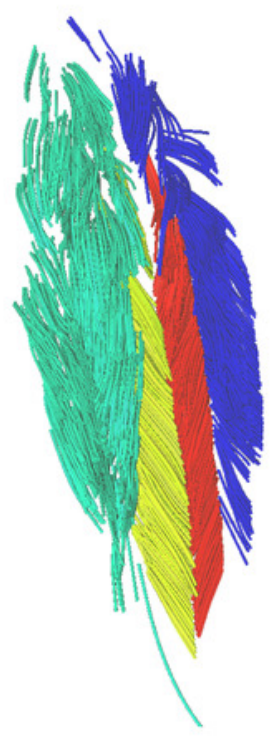




\section{Figure 2}

Anterior view of a 3D fascicle reconstruction of a cadaveric soleus muscle

The reconstruction was made through dissection and 3D digitisation. Note the resemblance with the in vivo reconstruction from DTI in Figure 1. To make the reconstruction, the tip of the digitiser was run along the course of a fascicle while recording the 3D coordinates of the tip. After digitisation of a fascicle, the fascicle (or a bundle of fascicles) was carefully removed. This process was repeated until all fascicles were removed. Colours indicate different compartments (same colours as in Fig. 1).

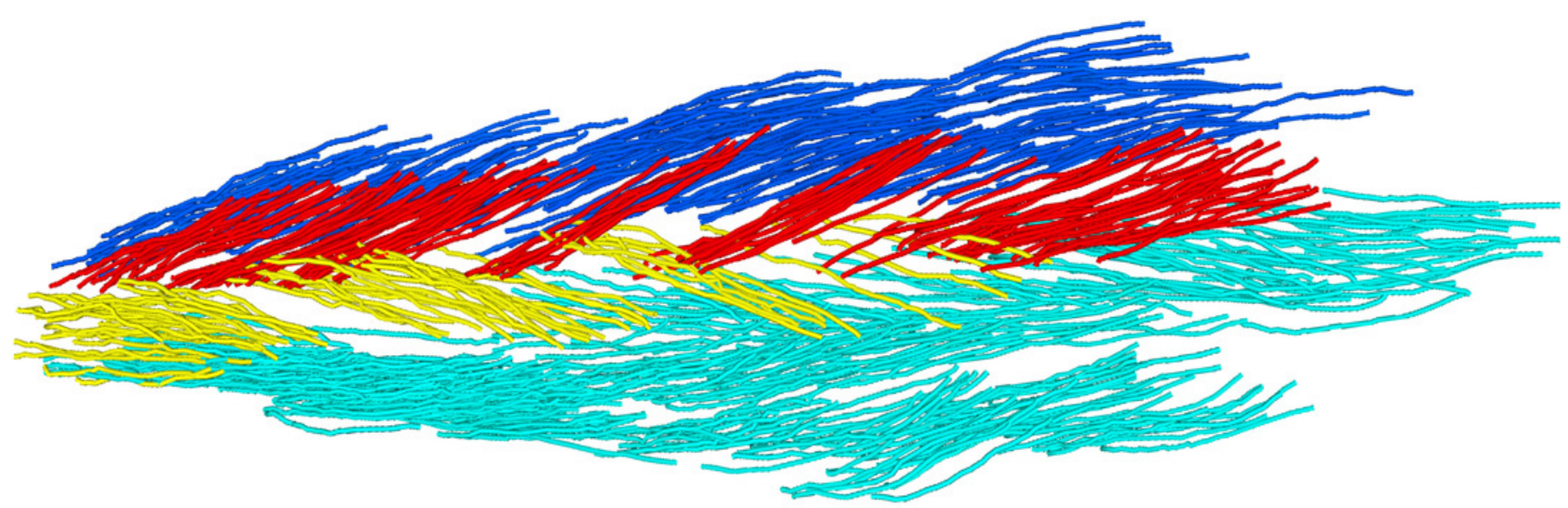




\section{Figure 3}

Relative volume of the four compartments of the human soleus

In all subjects the posterior compartments were substantially larger than the anterior compartments, but there was considerable inter-individual variation. The volume of a compartment is the mean of the volumes measured at the short and the long length. 


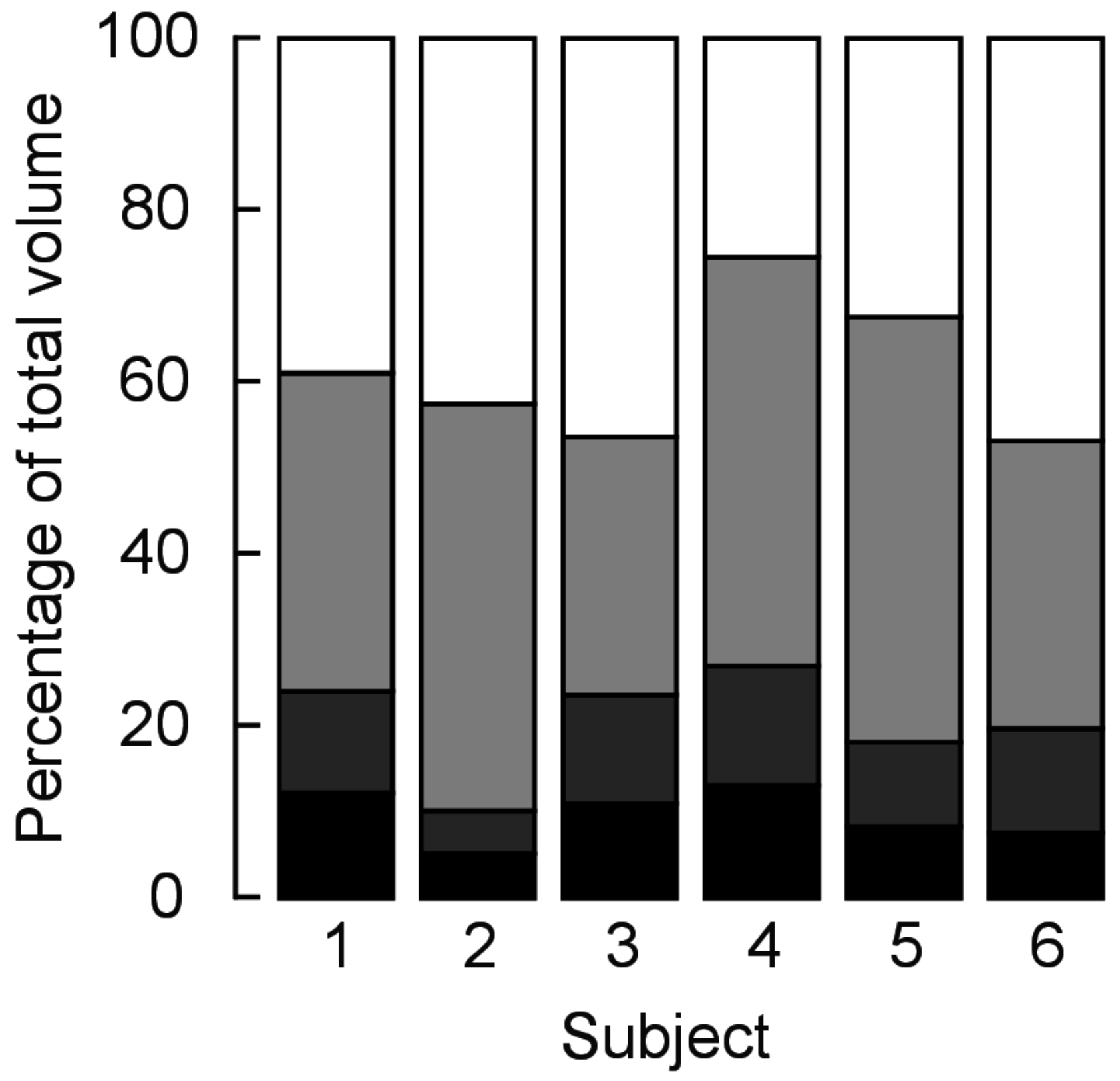

medial-anterior $\square$ medial-posterior
lateral-anterior $\square$ lateral-posterior 


\section{Figure 4}

Example of 3D reconstruction and muscle architecture measurements.

Anterior view of the soleus muscle of one subject with the muscle at a short (A) and a long length (B). Fascicles are coloured according to their length. Distribution of $(C)$ fascicle lengths (bin size $=2 \mathrm{~mm}$ ) and $(D)$ pennation angle (bin size $=2^{\circ}$ ) for all four compartments (same colours as in Fig. 1; see also inset in C). The solid and dashed lines present measurements obtained at the short and long muscle length, respectively. Fraction = fraction of total number of successfully reconstructed fascicles. 
A

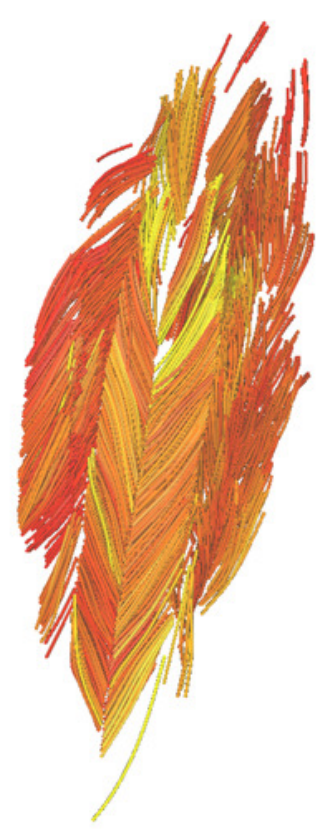

C

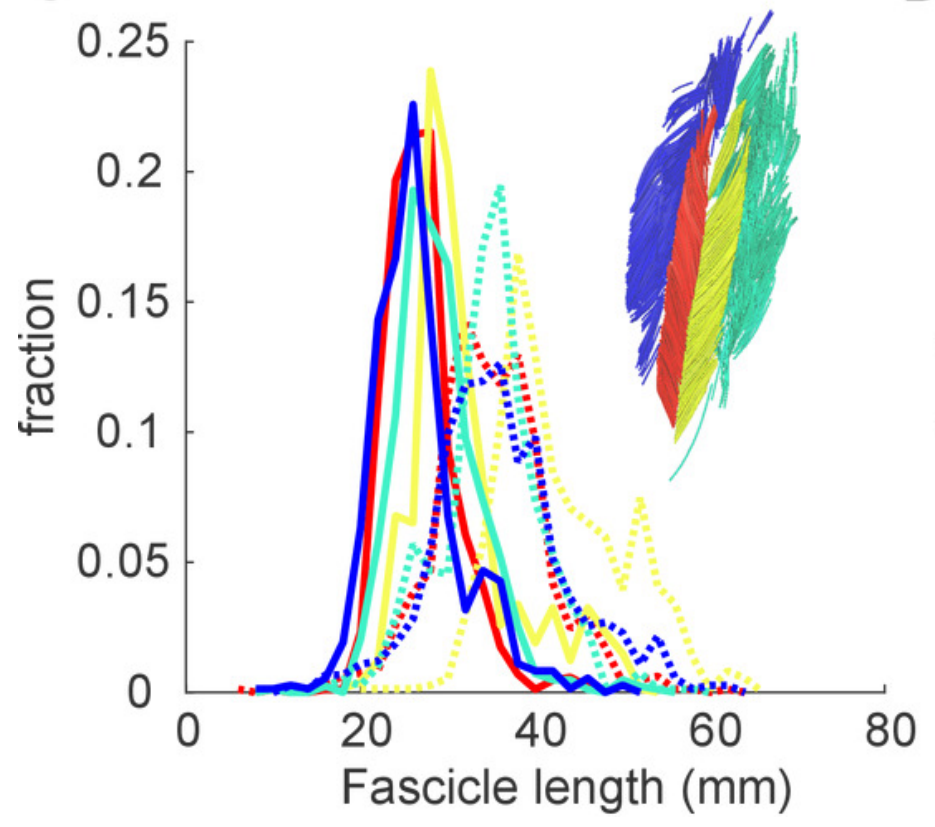

B

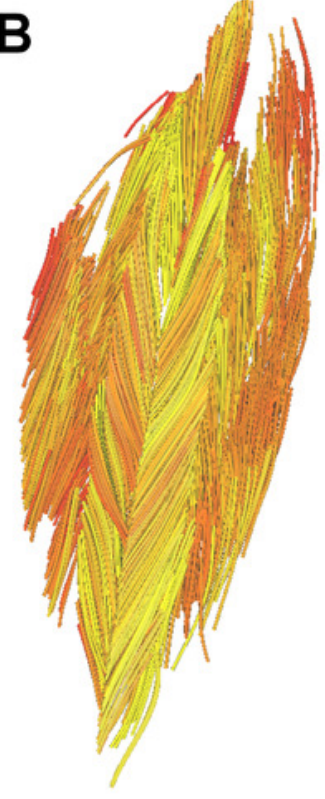

$50 \mathrm{~mm}$

D

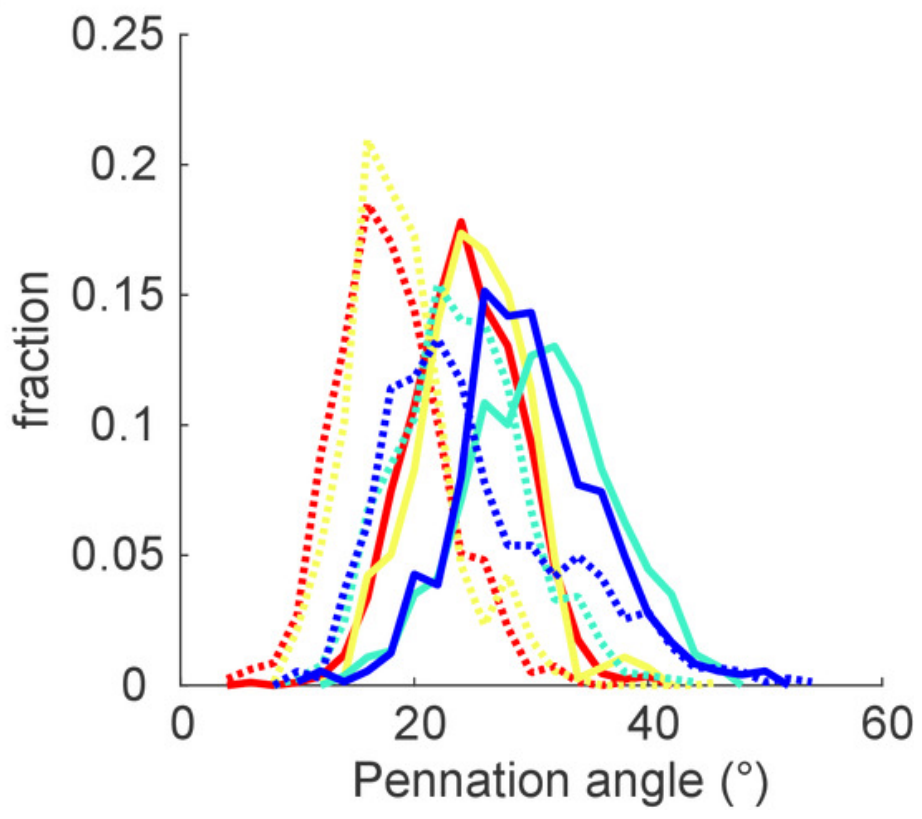


Figure 5

Muscle architecture of the soleus at the short muscle length.

(A) Fascicle length, (B) pennation angle and (C) curvature, grouped by muscle compartment. White squares and vertical bars indicate mean value \pm 1 SD over all subjects. Thin dashed lines connect the measurements made on one subject. Horizontal bars indicate statistically significant differences between compartments ( $* p<0.05, * * p<0.01, * * *<0.001)$. MA $=$ medial-anterior, $\mathrm{LA}=$ lateral-anterior, $\mathrm{MP}=$ medial-posterior, $\mathrm{LP}=$ lateral-posterior.
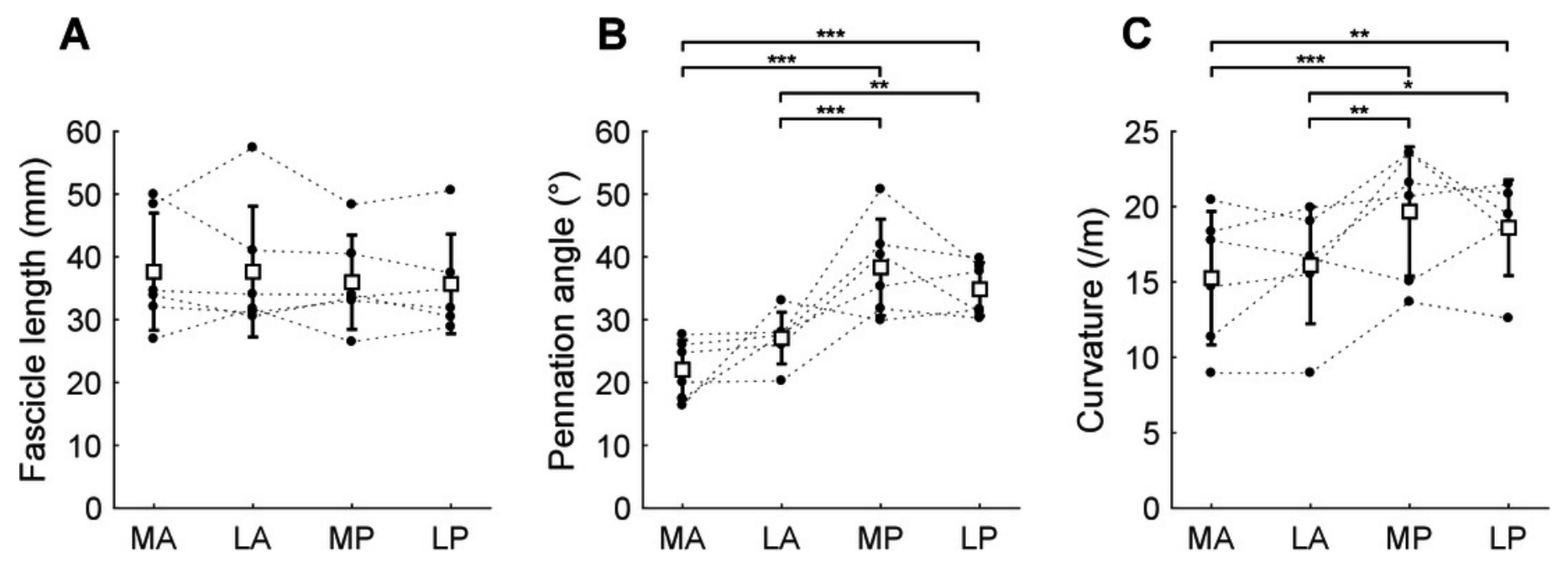
Figure 6

Change in architecture of the soleus with passive muscle lengthening.

(A) Fascicle length, (B) pennation angle and (C) curvature, grouped by muscle compartment. Values are parameter change per centimetre increase in muscle-tendon length. White squares and vertical bars indicate mean value \pm 1 SD over all subjects. Thin dashed lines connect the measurements made on one subject. Horizontal bars indicate statistically significant differences between compartments ( $* p<0.05, * * p<0.01, * * *<0.001)$. MA $=$ medial-anterior, $\mathrm{LA}=$ lateral-anterior, $\mathrm{MP}=$ medial-posterior, $\mathrm{LP}=$ lateral-posterior.

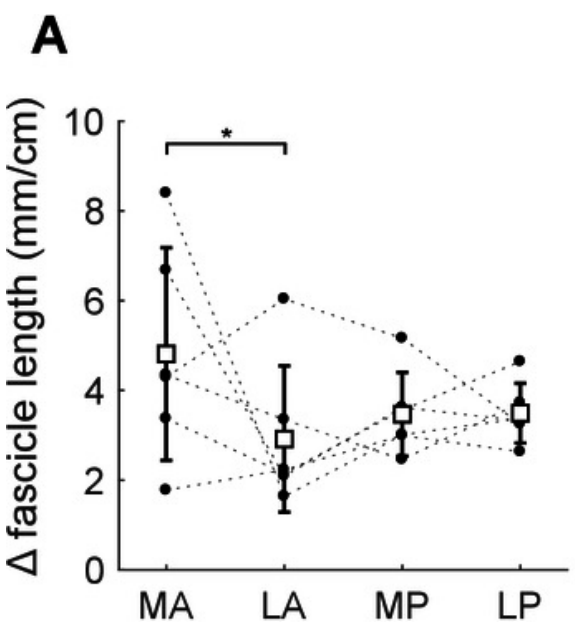

B

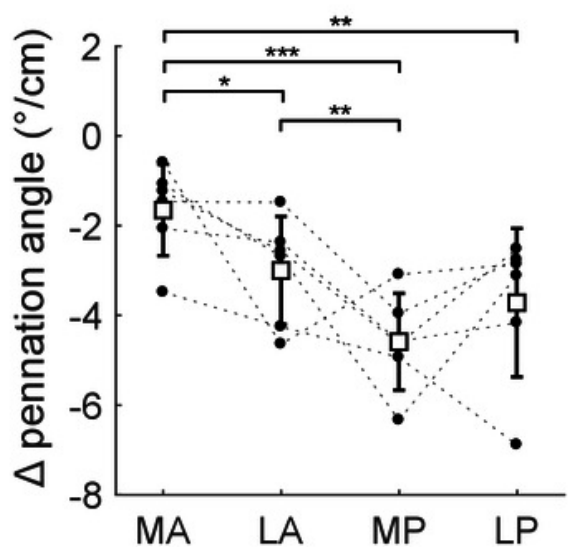

C

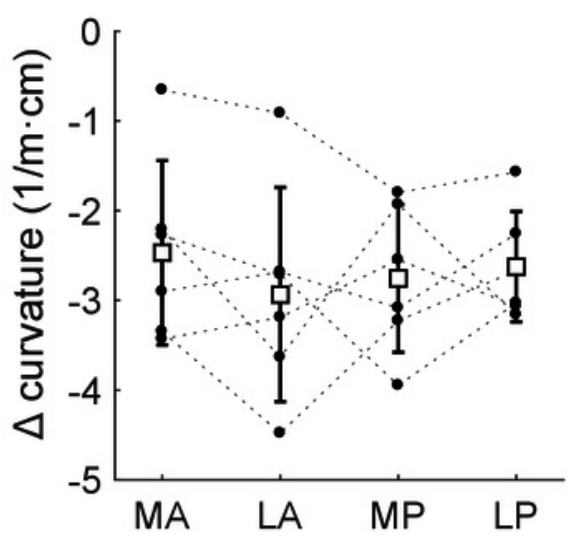


Table $\mathbf{1}$ (on next page)

Characteristics of participants and joint position 
1 Table 1

2 Characteristics of participants and joint positions

\begin{tabular}{|l|l|}
\hline Characteristic & Value \\
\hline Age (years) & $29.3 \pm 5.2$ \\
\hline Gender (M:F) & $3: 3$ \\
\hline Height (cm) & $168.0 \pm 6.8$ \\
\hline Weight (kg) & $62.9 \pm 4.1$ \\
\hline Shank length (cm) ${ }^{*}$ & $39.3 \pm 2.2$ \\
\hline Knee angle $\left(^{\circ}\right)$ & $20 \pm 5$ \\
\hline Ankle angle in 'short' position $\left(^{\circ}\right)^{\dagger}$ & $69 \pm 12$ \\
\hline Ankle angle in 'long' position $\left(^{\circ}\right)^{\dagger}$ & $108 \pm 7$ \\
\hline Change in muscle-tendon length $(\mathrm{mm})$ & $30.0 \pm 8.0$ \\
\hline
\end{tabular}

3 * Shank length was measured as the distance from the lateral femoral condyle to the middle of the

4 lateral malleolus.

5 t Ankle joint angle is the angle between the tibia and the sole of the foot, where $90^{\circ}$ is a neutral ankle

6 joint orientation and values below and above $90^{\circ}$ indicate plantarflexion and dorsiflexion, respectively. 


\section{Table 2 (on next page)}

Muscle architecture per soleus compartment at short muscle lengths, long muscle lengths and the change in architecture from short to long lengths (change = long short)

Values are means \pm SDs over all subjects of the average parameter value of all fascicles in a muscle compartment. For the whole muscle (last three rows), values are means \pm SDs over all subjects of the summed (for volume and PCSA) or average (for fascicle length, pennation and curvature) value over all compartments. 
Table 2

2 Muscle architecture per soleus compartment at short muscle lengths, long muscle lengths and the

3 change in architecture from short to long lengths (change = long - short). Values are means \pm SDs over all

4 subjects of the average parameter value of all fascicles in a muscle compartment. For the whole muscle

5 (last three rows), values are means \pm SDs over all subjects of the summed (for volume and PCSA) or

6 average (for fascicle length, pennation and curvature) value over all compartments.

\begin{tabular}{|l|l|c|c|c|c|c|}
\hline Compartment & Condition & $\begin{array}{c}\text { Volume } \\
\left(\mathbf{c m}^{3}\right)\end{array}$ & $\begin{array}{c}\text { PCSA } \\
\left(\mathbf{c m}^{2}\right)\end{array}$ & $\begin{array}{c}\text { Fascicle } \\
\text { length }(\mathbf{m m})\end{array}$ & $\begin{array}{c}\text { Pennation } \\
\text { angle } \mathbf{(}^{\circ}\end{array}$ & $\begin{array}{c}\text { Curvature } \\
(\mathbf{m})\end{array}$ \\
\hline $\begin{array}{l}\text { Medial- } \\
\text { anterior }\end{array}$ & Short & $33.5 \pm 10.1$ & $9.3 \pm 3.5$ & $37.7 \pm 9.3$ & $22.0 \pm 4.7$ & $15.3 \pm 4.4$ \\
\hline & Long & $33.5 \pm 11.0$ & $6.7 \pm 2.4$ & $51.9 \pm 13.3$ & $17.3 \pm 3.1$ & $7.9 \pm 2.0$ \\
\hline & Change & $0.0 \pm 2.1$ & $-2.6 \pm 1.8$ & $14.2 \pm 7.3$ & $-4.7 \pm 2.5$ & $-7.4 \pm 3.8$ \\
\hline $\begin{array}{l}\text { Lateral- } \\
\text { anterior }\end{array}$ & Short & $40.4 \pm 14.4$ & $10.9 \pm 4.1$ & $37.7 \pm 10.4$ & $27.1 \pm 4.1$ & $16.1 \pm 3.9$ \\
\hline & Long & $37.0 \pm 13.2$ & $8.0 \pm 2.3$ & $45.9 \pm 9.2$ & $18.6 \pm 1.8$ & $7.7 \pm 1.2$ \\
\hline $\begin{array}{l}\text { Medial- } \\
\text { posterior }\end{array}$ & Change & $-3.4 \pm 4.7$ & $-3.0 \pm 2.8$ & $8.3 \pm 3.7$ & $-8.5 \pm 2.6$ & $-8.4 \pm 3.1$ \\
\hline & Short & $146.6 \pm 29.1$ & $41.5 \pm 8.8$ & $36.0 \pm 7.5$ & $38.3 \pm 7.7$ & $19.7 \pm 4.3$ \\
\hline & Cong & $139.4 \pm 25.1$ & $30.7 \pm 5.1$ & $45.9 \pm 7.5$ & $24.5 \pm 3.7$ & $11.4 \pm 2.5$ \\
\hline $\begin{array}{l}\text { Lateral- } \\
\text { posterior }\end{array}$ & Short & $141.7 \pm 47.4$ & $40.1 \pm 12.5$ & $35.7 \pm 7.9$ & $34.9 \pm 4.3$ & $18.6 \pm 3.2$ \\
\hline & Long & $139.2 \pm 50.1$ & $29.9 \pm 7.8$ & $46.2 \pm 10.1$ & $24.2 \pm 2.4$ & $10.8 \pm 2.8$ \\
\hline & Change & $-2.5 \pm 5.4$ & $-10.2 \pm 5.6$ & $10.5 \pm 3.7$ & $-10.7 \pm 4.0$ & $-7.8 \pm 2.4$ \\
\hline $\begin{array}{l}\text { Whole- } \\
\text { muscle }\end{array}$ & Short & $362.2 \pm 59.2$ & $101.8 \pm 19.1$ & $36.8 \pm 8.4$ & $30.6 \pm 3.3$ & $17.4(3.6)$ \\
\hline & Long & $349.1 \pm 57.6$ & $75.2 \pm 8.0$ & $47.5 \pm 8.6$ & $21.1 \pm 1.9$ & $9.4 \pm 1.9$ \\
\hline & Change & $-13.1 \pm 9.7$ & $-26.6 \pm 11.5$ & $10.7 \pm 2.7$ & $-9.4 \pm 2.6$ & $-8.0 \pm 2.9$ \\
\hline
\end{tabular}

7 


\section{Table 3(on next page)}

Muscle architecture per soleus compartment of two human cadaveric soleus muscles measured by microdissection techniques.

The values represent the mean \pm SD of all fascicles that were digitised in that compartment. 


\section{Table 3}

2 Muscle architecture per soleus compartment of two human cadaveric soleus muscles measured by

3 microdissection techniques. The values represent the mean \pm SD of all fascicles that were digitised in

4 that compartment.

\begin{tabular}{|l|l|c|c|c|}
\hline & Compartment & $\mathbf{n}$ & Fascicle length $(\mathbf{m m})$ & Pennation angle $\left(^{\circ}\right)$ \\
\hline Muscle 1 & Medial-anterior & 102 & $37.9 \pm 4.9$ & $25.0 \pm 5.9$ \\
\hline & Lateral-anterior & 97 & $34.3 \pm 5.0$ & $28.8 \pm 10.3$ \\
\hline & Medial-posterior & 123 & $31.4 \pm 5.1$ & $19.6 \pm 4.7$ \\
\hline \multirow{2}{*}{ Muscle 2 } & Lateral-posterior & 68 & $27.9 \pm 7.1$ & $24.2 \pm 7.4$ \\
\hline & Medial-anterior & 90 & $50.9 \pm 4.8$ & $19.9 \pm 4.6$ \\
\hline & Lateral-anterior & 58 & $46.2 \pm 5.0$ & $18.0 \pm 4.4$ \\
\hline & Medial-posterior & 162 & $43.9 \pm 3.1$ & $16.8 \pm 3.1$ \\
\hline & Lateral-posterior & 294 & $42.0 \pm 4.8$ & $15.0 \pm 3.9$ \\
\hline
\end{tabular}

5 